\title{
Measuring the clustering of photometric quasars through blind mitigation of systematics
}

\author{
Boris Leistedt, Hiranya V. Peiris, Nina Roth \\ Department of Physics and Astronomy, \\ University College London, London WC1E 6BT, U.K. \\ boris.leistedt.11@ucl.ac.uk, h.peiris@ucl.ac.uk, n.roth@ucl.ac.uk
}

\begin{abstract}
We present accurate measurements of the large-scale clustering of photometric quasars from the Sloan Digital Sky Survey. These results, detailed in Leistedt \& Peiris (2014), rely on a novel technique to identify and treat systematics when measuring angular power spectra, using null-tests and analytical marginalisation. This approach can be used to maximise the extraction of information from current and future galaxy or quasar surveys. For example, it enables to robustly constrain primordial non-Gaussianity (PNG), which modifies the bias of galaxies and quasars on large scales - the most sensitive to observational systematics. The constraints on PNG obtained with the quasar power spectra are detailed in Leistedt, Peiris \& Roth (2014); these are the most stringent constraints to date obtained with a single tracer of the large-scale structure.
\end{abstract}

Keywords. quasars: general, cosmology: large-scale structure of universe, early universe.

\section{Motivation}

Quasars are bright, highly biased tracers of the large scale structure (LSS) of the universe, and are useful for testing cosmological models over large volumes and extended redshift ranges. In particular, photometric quasar surveys can be used to tightly constrain primordial non-Gaussianity (PNG) of local type, parameterised by $f_{\mathrm{NL}}$, which is predicted to enhance the bias of LSS tracers on large scales (e.g., Dalal et al. 2008; Matarrese \& Verde 2008; LoVerde et al. 2008). However, this requires accurate autoand cross-correlation power spectrum measurements, which are complicated by the presence of significant systematics in the data. These systematics can be intrinsic (e.g., dust extinction), observational (e.g., seeing, airmass), or instrumental (e.g., instrument calibration), and affect the properties of the data in complex ways, creating spurious spatial correlations in quasar catalogues. Previous studies of the clustering of quasars from the Sloan Digital Sky Survey (SDSS) exhibited power excesses on large and small scales (e.g., Myers et al. 2007), particularly significant in the DR6 and DR8 photometric quasar catalogues (Richards et al. 2009; Bovy et al. 2012). Cuts to the data were not sufficient to remove this excess power, apparently pointing to significant levels of PNG (Xia et al. 2009, 2011; Giannantonio et al. 2012; Karagiannis, Shanks \& Ross 2014; Giannantonio et al. 2014). However, recent work has demonstrated that the excess power was in fact due to systematics (Pullen \& Hirata 2013; Ho et al. 2013; Agarwal et al. 2014) and could be eliminated using mode projection (Leistedt et al. 2013). The latter consists of an analytical marginalisation of the spurious clustering using templates of systematics, and is a generalisation of the correction techniques introduced in Ross et al. (2011, 2012); Ho et al. (2012); Agarwal et al. (2014). 


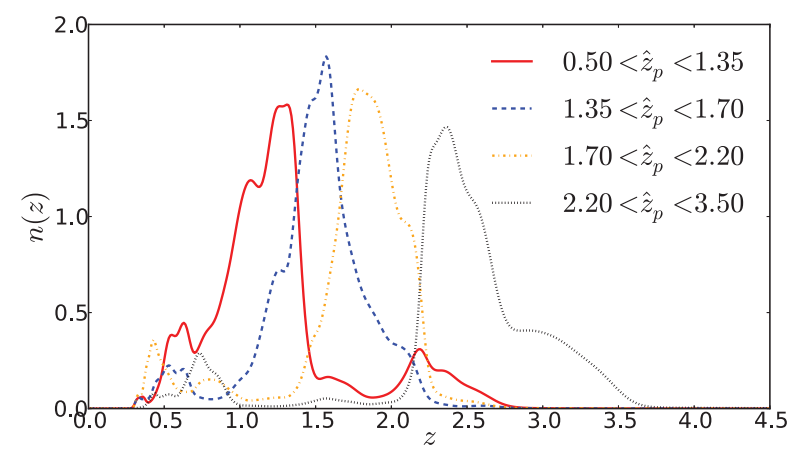

Figure 1. Estimated redshift distributions of the four quasar samples used in this analysis.

\section{Data samples}

We consider the XDQSOz catalogue of photometric quasars (Bovy et al. 2012), which contains quasar candidates selected from point sources detected in the SDSS DR8 imaging data, covering $\sim 10^{4} \mathrm{deg}^{2}$ of the southern and northern Galactic sky. We consider all objects with $\mathrm{P}_{\mathrm{QSO}}>0.8$, and exclude regions of the sky with $\mathrm{PSFWIDTH}_{i}>2.0, \mathrm{SCORE}>$ 0.6 , and $E(B-V)>0.08$, to remove the most dusty regions in the South Galactic Cap. $\mathrm{XDQSOz}$ also includes photometric redshift estimates $\hat{z}_{p}$, defined as the highest peak in the posterior distribution of the individual photo- $z$ estimates. We separate this catalogue into four samples by selecting objects with photometric redshifts $\hat{z}_{p}$ in top-hat windows ranging $[0.5,1.35],[1.35,1.7],[1.7,2.2]$ and $[2.2,3.5]$, which have comparable numbers of objects, and thus analogous shot noise. The redshift distributions of these samples, which are needed to produce theoretical predictions for the clustering of quasars, are shown in Figure 1, and were estimated by stacking the posterior distributions of the redshift of the individual objects.

\section{Power spectrum measurements}

We use a quadratic maximum likelihood estimator (QML) to simultaneously estimate the angular power spectra of these four quasar samples. The resolution of the data and the settings of the estimator are fixed to obtain accurate power spectra for $\ell \in[2,140]$ (smaller scales are dominated by shot noise), following the rules detailed in Leistedt et al. (2013). In particular, we estimate band powers in multipole bins $\Delta \ell=15$ and 21 .

We create a set of 220 templates of potential systematics, using external data (e.g., maps of extinction by dust and stellar density), and numerous calibration and observing condition quantities, presented in detail in Leistedt \& Peiris (2014). The red data points in Figure 2 show the estimated band-power spectra when projecting out the modes of this base set of templates ('basic mp'). The black lines denote the theoretical power spectra corresponding to a Planck $\Lambda$ CDM cosmology (Planck Collaboration 2013), $f_{\mathrm{NL}}=0$, and fiducial linear quasar bias $b(z)=1+[(1+z) / 2.5]^{5}$. The shaded band indicates the zone spanned by the theory curves when varying $-50<f_{\mathrm{NL}}<50$.

Projecting out these templates in the estimator is equivalent to marginalising their linear effect on the quasar maps, therefore removing their influence on the measured power spectra to first order. However, the latter still suffer from excess power on large scales when compared to the theoretical prediction. This could indicate significant nonlinear effects of the systematics, which cannot be addressed by marginalising over the base templates. 


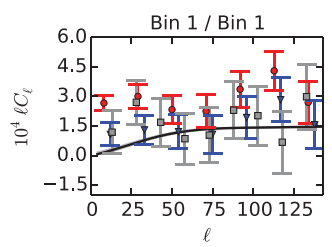

- Theory, $f_{\mathrm{NL}}=0$

$\square$ Theory, $-50<f_{\mathrm{NL}}<50$

호 호 Mask 2, $\Delta \ell=21$, basic $\mathrm{mp}$

亦重 Mask 2, $\Delta \ell=21$, ext. $\mathrm{mp}$ (null tests with $\Delta \ell=21$ )

호호 Mask 2, $\Delta \ell=15$, ext. $\mathrm{mp}$ (null tests with $\Delta \ell=15$ )
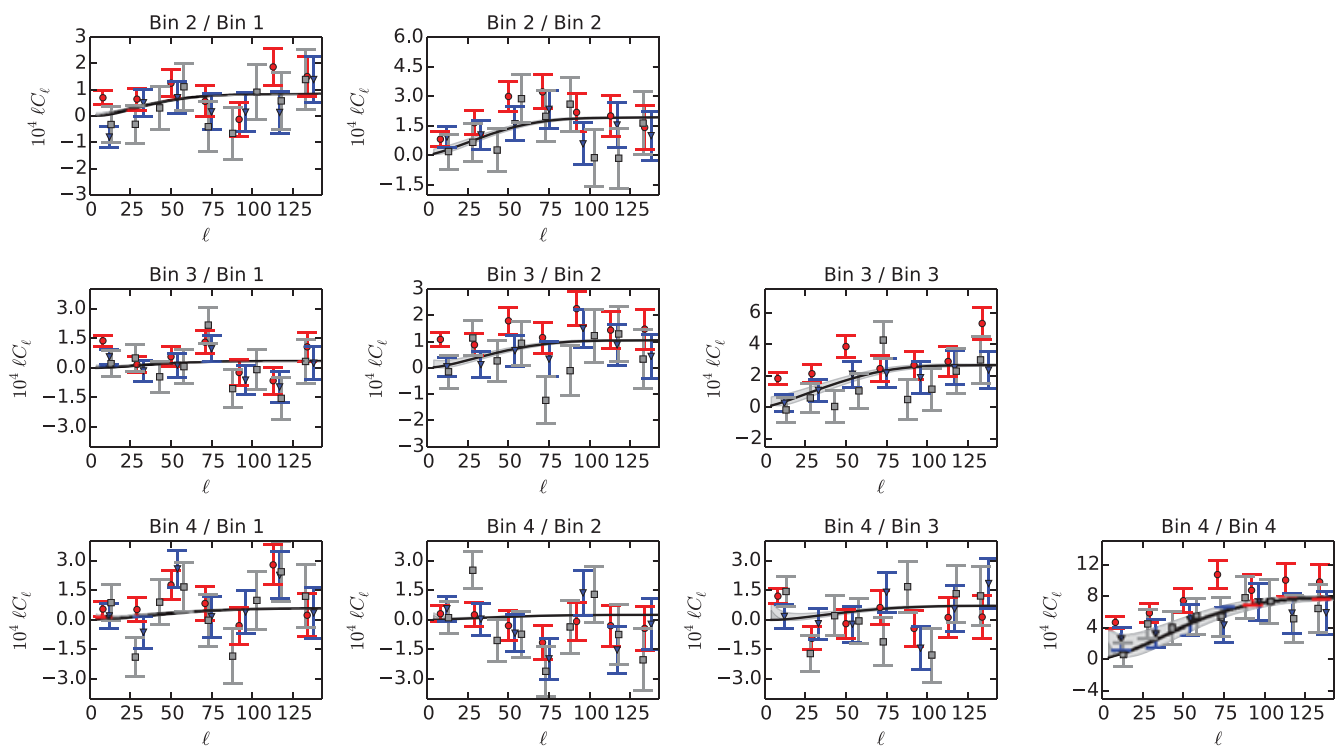

Figure 2. Angular power spectra of the four XDQSOz quasar samples. The effects of systematics are mitigated using either simple mode projection (linear contamination model of $\sim 200$ templates), or the novel extended mode projection approach (quadratic model with $\sim 20,000$ templates, decorrelated on the sky and cross-correlated with the data). See text for more details.

\section{Extended mode projection}

To enable a more complex treatment of systematics and cleaner power spectrum measurements, we exploit a novel technique of extended mode projection, introduced in Leistedt \& Peiris (2014). This involves having a more complete set of systematics templates but only projecting out the ones which are significantly correlated with the data. For this purpose, we complement the base set of systematics with pairs of products of templates, yielding a set of $\sim 20,000$ templates. We then decorrelate them on the sky, by applying a principal component analysis and discarding the modes with eigenvalues consistent with noise or numerical precision, leading to $\sim 3,700$ uncorrelated templates (replacing the $\sim 20,000$ input templates). We then cross-correlate each template with the four redshift samples in order to obtain cross-angular power spectra which can be used as null tests. We only marginalise (through mode projection) over the modes which fail the null tests, i.e., where the cross-power spectra are not consistent with zero, as measured by the reduced $\chi^{2}$ using a simple Gaussian likelihood. The blue and grey data points in Figure 2 show the results of this approach with two multipole resolutions $\Delta \ell=15$ and $\Delta \ell=21$. In both cases, the excess power due to systematics is eliminated, and the measurements are in good agreement with the theoretical predictions, while not showing further evidence for contamination by systematics (i.e., their effects lie within the statistical errors). 


\section{Outlook}

It was previously shown that effects of systematics on angular power spectrum measurements could be robustly marginalised through mode projection. However, quasar surveys are subject to strong non-linear systematics which require a more complicated treatment, for example involving non-linear contamination models with large numbers of templates. Extended mode projection can deal with this setting by decorrelating the templates and only projecting out the ones which are significantly correlated with the data. This approach is entirely based on mapping potential systematics and performing null tests with the data, and is therefore a robust way to handle systematics in a blind fashion. More details on extended mode projection and the power spectra of the XDQSOz data can be found in Leistedt \& Peiris (2014).

Figure 2 also demonstrates that the measured power spectra reach a sufficient precision and robustness to constrain PNG, which modifies the bias of quasars on large scales. The constraints on PNG from the XDQSOz power spectra are presented in Leistedt, Peiris \& Roth (2014), and are the most stringent constraints to date obtained with a single tracer of the large-scale structure. The power of the extended mode projection approach can be applied to extract the maximum information from future galaxy and quasar surveys, which will suffer from numerous observational systematics, most of which can be mapped on to the sky.

\section{References}

Agarwal, N., Ho, S., Myers, A. D., Seo, H.-J., Ross, A. J. et al. 2014, JCAP, 1404, 007, arXiv: 1309.2954

Bovy, J., et al. 2012, ApJ, 749, 41, arXiv:1105.3975

Dalal, N., Doré, O., Huterer, D., \& Shirokov, A. 2008, Phys. Rev. D., 77, 123514, arXiv: 0710.4560

Giannantonio, T., Crittenden, R., Nichol, R., \& Ross, A. J. 2012, MNRAS, 426, 2581, arXiv: 1209.2125

Giannantonio, T., Ross, A. J., Percival, W. J., Crittenden, R., Bacher, D., Kilbinger, M., Nichol, R., \& Weller, J. 2014, Phys. Rev. D, 89, 023511, arXiv:1303.1349

Ho, S., et al. 2013, arXiv preprint, arXiv:1311.2597

Ho, S., et al. 2012, ApJ, 761, 14, arXiv: 1201.2137

Karagiannis, D., Shanks, T., \& Ross, N. P. 2014, MNRAS, 441, 486, arXiv:1310.6716

Leistedt, B. \& Peiris, H. V. 2014, arXiv preprint, arXiv: 1404.6530

Leistedt B., Peiris H. V., Mortlock D. J., Benoit-Lévy A., \& Pontzen, A. 2013, MNRAS, 435 , 1857, arXiv: 1306.0005

Leistedt, B., \& Peiris, H. V., \& Roth, N. 2014, arXiv preprint, arXiv:1405.4315

LoVerde, M., Miller, A., Shandera, S., \& Verde, L. 2008, ApJ (Letters), 4, 14, arXiv:0711.4126

Matarrese, S. \& Verde, L. 2008, ApJ (Letters), 677, L77, arXiv:0801.4826

Myers, A. D., Brunner, R. J., Richards, G. T., Nichol, R. C., Schneider, D. P., \& Bahcall, N. A. 2007, ApJ, 658, 99, arXiv:arXiv:astro-ph/0612191

Planck Collaboration, 2013, arXiv preprint, arXiv:1303.5076

Pullen, A. R. \& Hirata, C. M. 2013, PASP, 125, 705, arXiv: 1212.4500

Richards, G. T., et al. 2009, ApJ (Supp.), 180, 67, arXiv:0809.3952

Ross, A. J., et al. 2011, MNRAS, 417, 1350, arXiv:1105.2320

Ross, A. J., et al. 2012, MNRAS, 424, 564, arXiv:1203.6499

Xia, J. -Q., Baccigalupi, C., Matarrese, S., Verde, L., \& Viel, M. 2011, J. Cosmol. Astropart. Phys., 8, 33, arXiv:1104.5015

Xia, J. -Q., Viel, M., Baccigalupi, C., \& Matarrese, S. 2009, J. Cosmol. Astropart. Phys., 9, 3, arXiv:0907.4753 